\title{
Pulmonary Embolism after Bilateral Upper Limb Surgeries: A Case Report and Review of Literature
}

\author{
Mujeeb Rohilla, Mohammad Waseem Beeharry, Gurudat Sisodia \\ Department of Trauma and Orthopaedics, Royal Surrey County Hospital, Guildford, UK \\ Email: m.w.beeharry@doctors.org.uk
}

How to cite this paper: Rohilla, M., Beeharry, M.W. and Sisodia, G. (2019) Pulmonary Embolism after Bilateral Upper Limb Surgeries: A Case Report and Review of Literature. Surgical Science, 10, 362-367. https://doi.org/10.4236/ss.2019.1010040

Received: August 27, 2019

Accepted: October 12, 2019

Published: October 15, 2019

Copyright $\odot 2019$ by author(s) and Scientific Research Publishing Inc. This work is licensed under the Creative Commons Attribution International License (CC BY 4.0).

http://creativecommons.org/licenses/by/4.0/

\begin{abstract}
Pulmonary embolism following upper limb surgery is a very rare complication. Here we present a unique case report of a 54-year-old young, fit and healthy, and non-smoker male who developed bilateral pulmonary embolism (PE) 26 days post-operatively with no associated upper or lower limb Deep Vein Thrombosis (DVT). This patient had minimal preoperative as well as intra-operative risk factors. He did not develop any thromboembolic symptoms until the 26th-day post-surgery when he developed sudden, sharp, pleuritic chest pain and laboured breathing. Initial baseline bloods showed a raised D-Dimer and the patient subsequently had a CT pulmonary angiogram that confirmed the presence of bilateral massive PEs. However, subsequent extremity ultrasound showed no upper or lower deep venous thrombosis. Thorough review of literature suggests that sudden development of PEs after upper limb surgery is possible, with a few cases being reported in the past. Symptoms such as sudden upper limb swelling, troubled breathing and systemic symptoms should be evaluated aggressively with a CT pulmonary angiogram, given the fact that an ultrasound of the extremities may be negative for deep venous thrombosis.
\end{abstract}

\section{Keywords}

CT Pulmonary Angiogram, D-Dimer, Deep Vein Thrombosis, Pulmonary Embolism, Ultrasound

\section{Introduction}

Pulmonary embolism (PE) following the upper limb surgeries is an extremely rare but potentially fatal complication. It is recognized as a major cause of mor- 
bidity and mortality in orthopaedic trauma patients. PE is a clinical risk with a reported incidence of 23 per 100,000 per year [1]. It is responsible for 200,000 deaths each year in the United States alone. It puts enormous financial costs on the health care system in the developed countries as well. As our healthcare system is increasingly emphasizing quality measures, it is critical for orthopaedic surgeons to understand the clinical factors that increase the risks of PE as well as Deep Vein Thrombosis (DVT) following orthopaedic procedures.

Traumatic injury results in significant physiologic changes that place the trauma patients at increased risk of venous thromboembolism (VTE), a term that comprises both DVTs and PEs. In trauma patients, the systemic inflammatory response results in a hypercoagulable state, with an increase of the serum levels of different cytokines (IL-6, IL-8, TNF-alpha etc.), which puts the patients at increased risks of developing DVT as well as PE post operatively. Hypercoagulability, along with endothelial injury and venous stasis, comprises Virchow's triad, which described the three broad categories of factors that are thought to contribute to thrombosis. Due to the frequent requirement of post-operative immobilization and protected weight-bearing following surgery, as well as improper mobilization in orthopaedics trauma surgery, all three conditions of Virchow's triad contribute to the development of DVTs and PEs.

So, any post-op patient with suspected PE must be taken seriously and should be seen and examined by the senior member of the team.

\section{Case Report}

A 54 year old gentleman, previously fit and healthy, a non-smoker, was brought into the Royal Surrey County Hospital by ambulance as a trauma call following a road traffic collision of his motorcycle with a car. He was an independent individual with no past medical history, no allergies and no regular medications. Primary survey was carried out as per the Advanced Trauma Life Support (ATLS) protocol. The patient was alert, responsive and orientated with bilaterally equal pupils and reacting to light with a GCS of 15/15. Cervical spine was stabilized using head blocks and all the vitals were maintained.

Secondary survey assessment revealed no head injury, no cervical spine tenderness, no clavicular tenderness, no chest wall bruising or deformity, abdomen was soft and non-tender, no pelvic motion tenderness and all 12 cranial nerves were intact. Examination of the limbs revealed that the left middle finger was amputated through the distal phalanx with a mallet deformity (Figure 1). The wound was washed in $250 \mathrm{mls}$ saline and dressed with Jelonet gauze dressing. The right distal radius showed a dinner fork deformity with preserved neurovascular status (Figure 2). This was reduced under local anaesthesia with $1 \%$ lidocaine block and placed in a below elbow back-slab. Post manipulation X-Ray revealed that the reduction was evident and in an acceptable position. There was a wound on the dorsum of the right 2 nd/3rd metacarpophalangeal (MCPJ); which was washed with $250 \mathrm{ml}$ of normal saline. The right knee was tender at 


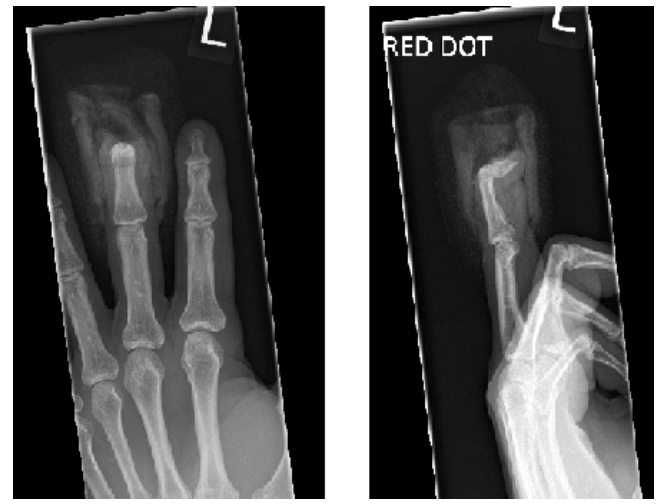

Figure 1. XR left hand: Amputation through the distal phalanx of the middle finger.

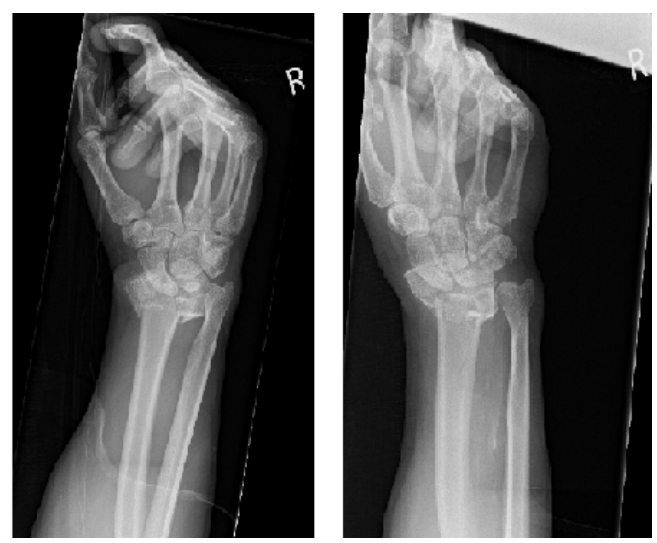

Figure 2. X-Ray right wrist (pre ORIF)—showing a displaced and comminuted intra-articular fracture of the distal radius.

the right medial femoral condyle and there was a laceration on the anterior aspect of knee. On clinical examination there was no un-stability found and all the tendons were intact. After washing the knee laceration with $0.9 \%$ of normal saline in the A\&E setting, the laceration was covered with Jelonet gauze dressing and a hinged knee brace was applied with full range of motion.

Trauma CT of the head and cervical spine showed no evidence of acute intra-cranial haemorrhage or any acute fractures. CT of the thorax, abdomen and pelvis showed no haemo-pneumothorax, no mediastinal haematoma or any overt diaphragmatic injury. There was, however, a non-displaced fracture of the medial right clavicle.

On the very next morning, after his case discussion at the trauma meeting together with the plastic surgeons, the patient was taken to the operation theatre. Open Reduction and Internal Fixation (ORIF) of the right distal radius fracture was performed by the orthopods (Figure 3). The distal phalynx of the left middle finger was amputated and the wound was covered with V-Y plasty. All the lacerations were washed with $0.9 \%$ normal saline and dressed with sterile dressings. Post-operatively, the patient made an unremarkable recovery and he was discharged the next day with a view to an outpatient appointment in 10 days time. 
On the 26th day post-surgery, the patient presented to A\&E with a complaint of right flank pain, shortness of breath and pleuritic chest pain. He was seen and examined by the junior A\&E doctor and was discharged on painkillers. However, due to his worsening shortness of breath and pleuritic chest pain, he re-presented A\&E the following day. His oxygen saturation was fluctuating between $88 \%-92 \%$ on room air. A blood test showed a raised D-dimer level of $2940 \mathrm{ng} / \mathrm{ml}$. He was taken for a CT pulmonary angiogram, with appearances suggesting large bilateral pulmonary emboli with no evidence of acute right heart strain (Figure 4). He was initially started on treatment dose of low molecular weight heparin for a duration of 5 days with the involvement of haematologist and was subsequently switched to Apixaban $5 \mathrm{mg}$ BD on discharge once his condition had stabilised and his care was transferred with a further follow up appointment with thehaematology service for pulmonary embolism.
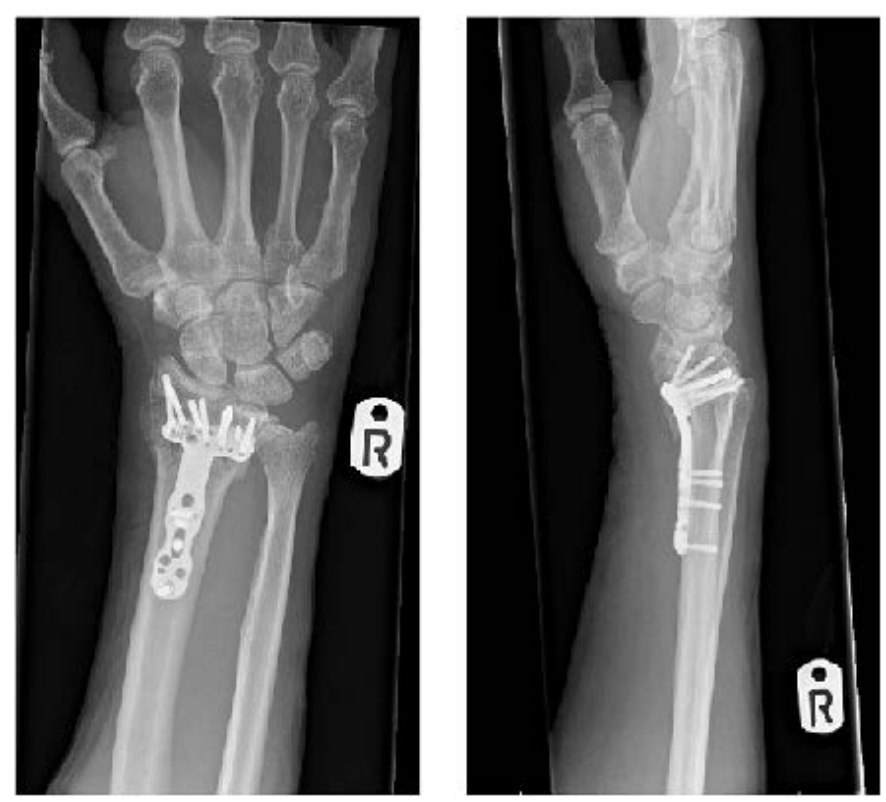

Figure 3. X-Ray right wrist (post ORIF) with plate and screws.

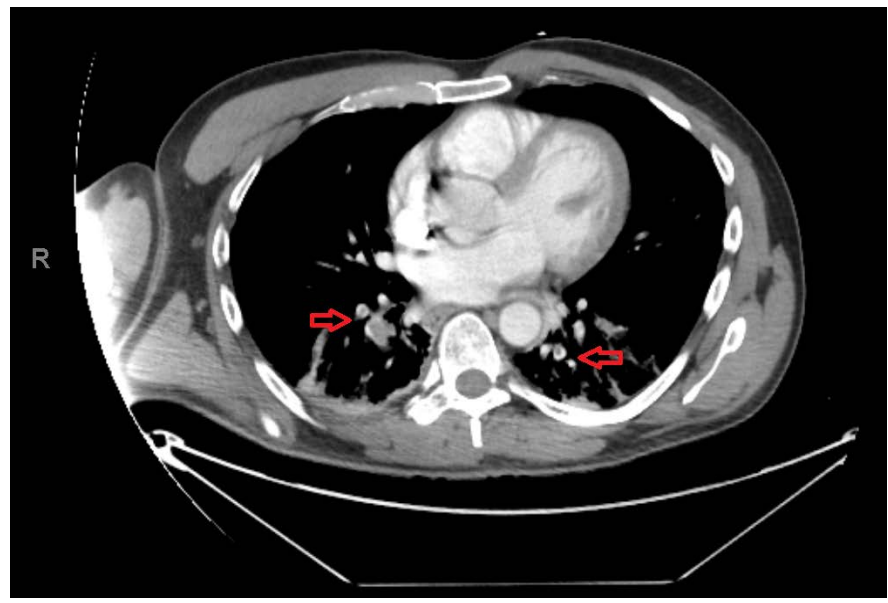

Figure 4. Bilateral pulmonary emboli showing no evidence of right heart strain. 


\section{Discussion}

Patients who undergo operations of the upper extremities and who are able to get out of bed and mobilise soon after, no aggressive prophylactic treatment are recommended [2] [3]. Moreover, there are no common prophylactic guidelines for pulmonary thromboembolism after operations of the upper extremities.

Pulmonary emboli that develop after upper limb surgery arise mainly from either the ipsilateral axillary subclavian venous system or from the lower extremities [4]. General risk factors for DVT following upper limb surgeries which are documented in the literature include the lateral decubitus position, prolonged surgical time, use of an inter-scalene nerve block, increasing age, positive family history of thromboembolism, smoking, obesity and diabetes mellitus. Surgical risk factors include venous irritation or compression, subcutaneous oedema and inadequate positioning of the upper limb. Medical risk factors for VTE in upper and lower extremities include varicosities, obesity, smoking, diabetes mellitus, rheumatoid arthritis, ischemic heart disease, neoplasia and venous stasis.

The National Institute for Health and Care Excellence (NICE) guidelines states that the following patients should be treated with anticoagulants: patients with active cancer or undergoing cancer treatment, age over 60 years, critical care admission, dehydration, known thrombophilias, obesity (body mass index over $30 \mathrm{~kg} / \mathrm{m}^{2}$ ), one or more significant medical comorbidities (for example: heart disease; metabolic, endocrine or respiratory pathologies; acute infectious diseases; inflammatory conditions), personal history or first-degree relative with a history of venous thromboembolism, hormone replacement therapy, oestrogen-containing contraceptive therapy, or varicose veins with phlebitis [5]. However, the prophylactic treatment for thromboembolism has been differently conducted according to the doctor in charge.

Given the wide variety of symptoms and presentation, we feel that PE cannot be diagnosed on the basis of history and examination alone. While ultrasound with venous imaging is portable and relatively inexpensive and has few contraindications, it is suitable for diagnosing DVT but certainly not PE. It is also operator dependent and less accurate for certain DVT formation, and false negative results may be obtained in the presence of calf DVT, proximal DVT in asymptomatic patients or in the presence of thrombosed duplicated venous segment [6]. In this case, CT pulmonary angiogram has been associated with greater than $95 \%$ sensitivity and specificity and has a greater sensitivity for emboli in the main, lobar or segmental pulmonary arteries due to its ability to define neurovascular structure such as lympdadenopathy, lung tumours, and other parenchymal abnormalities [7]. Ultrasound has been reported to have a negative predictive value of $97 \%$ to $98 \%$ while CT has detected all clinically relevant PE and a large number of alternative diagnosis [6]. As a result, any patient presenting with upper limb swelling, constitutional symptoms, difficulty in breathing and in the presence of derange baseline blood markers, we would recommend to 
do a spiral CT pulmonary angiogram to evaluate PE first and if it is negative then we would recommend to do upper extremity ultrasound to evaluate for DVT.

\section{Conclusion}

This patient does not have any "strong" risk factors as described in the NICE guidelines on the prevention of thromboembolism for the patients going for Orthopaedic surgery. In general, upper extremity surgery carries a very low risk of developing pulmonary embolism. Patients who are post-op and who present to A\&E with some symptoms like laboured breathing, pleuritic chest pain or confusion etc. should not be seen by the junior member of staff or at least they must consult their senior colleagues before discharging them. If in doubt or any suspicious case, a CT pulmonary angiogram must be considered in order to rule out PE before discharging them.

\section{Conflicts of Interest}

The authors declare no conflicts of interest regarding the publication of this paper.

\section{References}

[1] Perrier, A. (1997) Noninvasive Diagnosis of Pulmonary Embolism. Heamatologica, 82, 328-331.

[2] Kearon, C., Akl, E.A., Comerota, A.J., Prandoni, P., Bounameaux, H., Goldhaber, S.Z., Nelson, M.E., Wells, P.S., Gould, M.K., Dentali, F., Crowther, M. and Kahn, S.R. (2012) American College of Chest Physicians. Antithrombotic Therapy and Prevention of Thrombosis, 9th ed: American College of Chest Physicians Evidence-Based Clinical Practice Guidelines. Chest, 141, e419S-e496S.

https://doi.org/10.1378/chest.11-2301

[3] Nakamura, K., Tachibana, S., Fuji, T., Akagi, M., Abe, Y., Ikeda, N., Ishii, S., Kobayashi, S., Sato, T., Nakamura, S., Higuchi, F., Fujita, S. and Yanagimoto, S. (2008) Japanese Orthopaedic Association (JOA) Clinical Practice Guideline on the Prevention of Venous Thromboembolism in Patients Undergoing Orthopaedic Treatments. 1. Nankodo, Tokyo. (In Japanese)

[4] Willis, A.A., Warren, R.F., Craig, E.V., et al. (2009) Deep Venous Thrombosis after Reconstructive Shoulder Arthroplasty: A Perspective Observational Study. Journal of Shoulder and Elbow Surgery, 18, 100-106. https://doi.org/10.1016/j.jse.2008.07.011

[5] National Institute for Health and Clinical Excellence (2007) Reducing the Risk of Venous Thromboembolism (Deep Vein Thrombosis and Pulmonary Embolism) in Inpatients Undergoing Surgery. NICE Clinical Guideline, No-46.

[6] Tapson, V.F., Carroll, B.A., Davidson, B.L., et al. (1999) The Diagnostic Approach to Acute Venous Thromboembolism. Clinical Practice Guidelines. American Journal of Respiratory and Critical Care Medicine, 160, 1043-1066.

https://doi.org/10.1164/ajrccm.160.3.16030

[7] Michiels, J.J., Gadisseur, A., van der Planken M., et al. (2005) A Critical Appraisal of Non-Invasive Diagnosis and Exclusion of Deep Vein Thrombosis and Pulmonary Embolism in Outpatients with Suspected Deep Vein Thrombosis and Pulmonary Embolism: How Many Test Do We Need? International Angiology, 24, 27-39. 